\title{
How the Covid-19 pandemic will end in 2022 and why we still need adapted vaccines
}

Dr. Carolina Diamandis ${ }^{1}$, Adrian Tudor ${ }^{1}$, Jonathan Feldman $^{1}$, and Olga Ivanova ${ }^{1}$

${ }^{1}$ Affiliation not available

January 13, 2022

\section{Hosted file}

Sars-CoV-2 end of pandemic phase.pdf available at https://authorea.com/users/410930/articles/ 552653-how-the-covid-19-pandemic-will-end-in-2022-and-why-we-still-need-adaptedvaccines 